\title{
Inactivation efficiency of slightly acidic electrolyzed water against microbes on facility surfaces in a disinfection channel
}

\author{
Zang Yitian ${ }^{1}$, Li Baoming ${ }^{2}$, Shi Zhengxiang ${ }^{2}$, Sheng Xiaowei ${ }^{1}$, \\ Wu Hongxiang ${ }^{1}$, Shu Dengqun ${ }^{1 *}$ \\ (1. College of Animal Science and Technology, Jiangxi Agricultural University, Nanchang 330045, China; 2. Key Laboratory of Structure \\ and Environment in Agricultural Engineering, Ministry of Agriculture, China Agricultural University, Beijing 100083, China)
}

\begin{abstract}
Slightly acidic electrolyzed water (SAEW, pH 6.0-6.5) is an ideal and environmentally-friendly disinfectant, which was used to prevent and control bacterial infections on farms. This work aims to investigate the inactivation effectiveness of SAEW in inactivating microbes in a disinfection channel. The bactericidal efficiency of SAEW on equipment surfaces was compared to two commercial disinfectants, Kuei A bromide solution (KAS, 5:1000 v/v) and Glutaraldehyde solution (GS, $5: 1000 \mathrm{v} / \mathrm{v}$ ). The disinfection effectiveness of SAEW in inactivating Salmonella enteritidis (S. enteritidis) on equipment surfaces in the disinfection channel was evaluated, and a model was developed using multiple linear regression analysis. Results indicated that SAEW was significantly $(p<0.05)$ more efficient than KAS and GS on kits and clothing in the disinfection channel at $1 \mathrm{~min}$. The SAEW did not contribute as aggressively to respiratory difficulty as KAS and GS. Maximum reductions of $2.362 \log _{10} \mathrm{CFU} / \mathrm{cm}^{2}, 2.613 \log _{10} \mathrm{CFU} / \mathrm{cm}^{2}$ and $2.359 \log _{10} \mathrm{CFU} / \mathrm{cm}^{2}$ for Salmonella enteritidis were obtained from clothing surfaces, iron materials, and kits treated with SAEW for $2.5 \mathrm{~min}$ at a chlorine concentration of $220 \mathrm{mg} / \mathrm{L}$. Moreover, the established model had a good fit-quantified by the determination coefficient $R^{2}(0.939)$ and a lack of fit test $(p>0.05)$. In addition, available chlorine concentration (ACC) was an important factor than other factors, and the inactivation efficiency of Salmonella enteritidis sprayed by SAEW treatment was different between iron materials, kits and clothing surfaces (iron > kit > clothing).
\end{abstract}

Keywords: slightly acidic electrolyzed water, disinfection channel, S. enteritidis, disinfection, bacterial infection, prevention and control, livestock farm

DOI: $10.25165 /$ j.ijabe.20171006.2848

Citation: Zang Y T, Li B M, Shi Z X, Sheng X W, Wu H X, Shu D Q. Inactivation efficiency of slightly acidic electrolyzed water against microbes on facility surfaces in a disinfection channel. Int J Agric \& Biol Eng, 2017; 10(6): 23-30.

\section{Introduction}

Disinfection is one of the principal strategies which was used to reduce the spread of pathogenic microorganisms, especially in the disinfection channel ${ }^{[1,2]}$. The disinfection channel, located at the entrance of farms,

Received date: 2016-09-12 Accepted date: 2017-05-10

Biographies: Zang Yitian, $\mathrm{PhD}$, research interests: environmental control techniques for animal production operations, Email: zangyitian1@126.com; Li Baoming, PhD, Professor, research interests: agricultural biological environment and energy engineering, Email: libm@cau.edu.cn; Shi Zhengxiang, PhD, Professor, research interests: animal environmental control, Email: shizhx@cau.edu.cn; Sheng Xiaowei, Master, research interests: exists to prevent the introduction and spread of infections from foreign personnel and equipment ${ }^{[3]}$. Objects such as clothing, iron materials, and drug kits can be easily contaminated by pathogenic microorganisms like $S$. enteritidis, one of the most frequent causes of avian colibacillosis $^{[4-6]}$. Poor hygiene has been identified as a

environmental control techniques for animal production, Email: 1120947790@qq.com; Wu Hongxiang, Master, research interests: environmental control techniques for animal production, Email: 2014035038@qq.com.

*Corresponding author: Shu Dengqun, $\mathrm{PhD}$, Professor, research interests: animal environmental control. College of Animal Science and Technology, Jiangxi Agricultural University, Jiangxi 330000, China. Tel/Fax: +86-791-83813503, Email: 13607047771@163.com. 
potential route of transmission for disease ${ }^{[7-9]}$, and it is imperative to disinfect materials that enter the farm, thus reducing the risk of pathogen contamination. However, potential toxic, corrosive, or volatile problems have arisen from the use of chemical disinfectants ${ }^{[10,11]}$. Therefore, it is important to develop an effective disinfectant with high efficacy and little harmful residue.

Slightly acidic electrolyzed water (SAEW) is produced by electrolysis of dilute hydrochloric acid in a chamber without a membrane at a $\mathrm{pH}$ of 5.0 to 6.5. The chamber contains a high concentration of hypochlorous acid, which has strong antimicrobial activity against many pathogens ${ }^{[12,13]}$. Relative to other disinfectants, SAEW has the extra advantage of reduced corrosion of surfaces. SAEW also minimizes human health and safety issues from $\mathrm{Cl}_{2}$ off-gassing ${ }^{[15-17]}$. It is the most environmentally friendly potential alternative to broad-spectrum microbial decontaminants ${ }^{[18]}$. Several studies have demonstrated that SAEW can be used as a disinfectant in the livestock industry ${ }^{[15,19]}$. Hao et al. ${ }^{[20]}$ reported that SAEW with an available chlorine concentration (ACC) of $300 \mathrm{mg} / \mathrm{L}$ resulted in a significant $(p<0.05)$ reduction in microbes on the walls, railings and floor of swine barns after flushing disinfection. Hao et al. $^{[21]}$ also indicated that treatment of floors, walls, feed troughs and water pipes with SAEW at ACC of $250 \mathrm{mg} / \mathrm{L}$ can significantly $(p<0.05)$ reduce bacteria in a layer house. However, little work has been done on the implications of spraying SAEW in the disinfection channel to combat microbes.

The objectives in this study firstly were to compare the bactericidal efficiency of SAEW and two other commercial disinfectants when used on clothing and kit surfaces. The other two disinfectants were Kuei A bromide solution (KAS) and Glutaraldehyde solution (GS), both of which are routinely applied in the disinfection channel. The second purpose was to build a mathematical model of SAEW which can be adopted in inactivating S. enteritidis on clothing surfaces, iron materials, and kits in the disinfection channel. The model was built as a function of treatment time and ACC using multivariate regression analysis.

\section{Materials and methods}

\subsection{Experimental disinfection channel}

The experiments were performed in a disinfection channel located in the city of Hebei, northeast of China from October to November of 2014. This facility with dimensions of $6.5 \mathrm{~m} \times 1 \mathrm{~m} \times 3 \mathrm{~m}$ (length $\times$ width $\times$ height) contained two automatic sprayers (droplets: 80-90 $\mu \mathrm{m}$ ), an incandescent lamp and a rubber mats. The automatic sprayers and incandescent lamp were both located on the south wall and the rubber mats were laid on the ground. Moreover, the two automatic sprayers connecting with two plastic buckets $(50$ L) were controlled by a disinfection automatic controller (Saint-fun environment technology Co., Qingdao China), and the height of the two automatic sprayers were both $2 \mathrm{~m}$.

\subsection{Bacterial cultures}

The strains of $S$. enteritidis (CVCC 2184) used in this work were from the China Veterinary Culture Collection (CVCC, Beijing, China). Stock cultures of pathogen were transferred into tryptic soy broth (TSB, Beijing Land Bridge Technology Company Ltd., Beijing, China) and incubated for $24 \mathrm{~h}$ at $35^{\circ} \mathrm{C}$. Following incubation, a $10 \mathrm{~mL}$ of culture was pooled into a sterile centrifuge tube and centrifugated at $3000 \times \mathrm{g}, 4^{\circ} \mathrm{C}$ for $10 \mathrm{~min}$. The supernatant was decanted, and the pellets were re-suspended in $10 \mathrm{~mL}$ of sterilized $0.85 \% \mathrm{NaCl}$ solution, washed three times and re-suspended in $10 \mathrm{~mL}$ of the same solution, to obtain a final cell concentration of about $10^{7} \mathrm{CFU} / \mathrm{mL}$ to $10^{8} \mathrm{CFU} / \mathrm{mL}$. The bacterial population in each culture was confirmed by plating $0.1 \mathrm{~mL}$ portions of appropriately diluted culture on tryptic soy agar (TSA, Beijing Land Bridge Technology Company Ltd., Beijing, China) plates and incubating the plates at $35^{\circ} \mathrm{C}$ for $24 \mathrm{~h}$.

\subsection{Preparation of disinfectants}

Slightly acidic electrolyzed water was produced using a non-membrane generator (Ruiande Biosafety Technology Co., Ltd., Beijing, China) to electrolyzing $\mathrm{NaCl}(1 \mathrm{~g} / \mathrm{L})$ containing $\mathrm{HCl}(100 \mu / \mathrm{L})$ solution. SAEW with a $\mathrm{pH}$ of 6.15-6.50, an oxidation-reduction potential (ORP) of $974 \mathrm{mV}$ to $989 \mathrm{mV}$, and different ACCs (Table 1) was produced by the SAEW generator. The physicochemical properties of SAEW were measured 
before use. The $\mathrm{pH}$ and ORP values were measured using a dual scale pH/ORP metre (CON60, Trans-Wiggens, Singapore) with a pH electrode (PE02; range 0.00 to 14.00) and an ORP electrode (ORP06; range -999 to $+999 \mathrm{mV}$ ). The ACC was determined using a digital chlorine test system (RC-2Z, Kasahara Chemical Instruments Co., Saitama, Japan). The detection range was 0 to $320 \mathrm{mg} / \mathrm{L}$. KAS (Shenyang Shengbao Biological technology Co., LTD., Shenyang, China) and Glutaraldehyde solution (GS, Beijing depot washing disinfection products Co., LTD., Beijing, China) were purchased from commercial suppliers. The solutions were placed into two plastic buckets of the two automatic sprayers before the experiment.

Table 1 Treatment conditions of the sample with kit, iron and clothes

\begin{tabular}{|c|c|}
\hline Time/min & $\mathrm{ACC} / \mathrm{mg} \cdot \mathrm{L}^{-1}$ \\
\hline 0.5 & 63 \\
\hline 0.5 & 108 \\
\hline 0.5 & 140 \\
\hline 0.5 & 200 \\
\hline 0.5 & 220 \\
\hline 1 & 63 \\
\hline 1 & 108 \\
\hline 1 & 140 \\
\hline 1 & 200 \\
\hline 1 & 220 \\
\hline 1.5 & 63 \\
\hline 1.5 & 108 \\
\hline 1.5 & 140 \\
\hline 1.5 & 200 \\
\hline 1.5 & 220 \\
\hline 2 & 63 \\
\hline 2 & 108 \\
\hline 2 & 140 \\
\hline 2 & 200 \\
\hline 2 & 220 \\
\hline 2.5 & 63 \\
\hline 2.5 & 108 \\
\hline 2.5 & 140 \\
\hline 2.5 & 200 \\
\hline 2.5 & 220 \\
\hline
\end{tabular}

\subsection{Comparison of the disinfectants}

The comparartive experiments were performed by 3 breeder (on the farm) clothings and 3 veterinary medicine kits (Jindanduo, Zhengda biological technology Co., LTD., Zhengzhou, China) sprayed with SAEW (ACC, $220 \mathrm{mg} / \mathrm{L}), \operatorname{KAS}(5: 1000 \mathrm{v} / \mathrm{v})$ and GS (5:1000 v/v) for $1 \mathrm{~min}$ in disinfection channel, respectively. The colony-forming units (CFU) of microorganisms on the breeder clothing surfaces and veterinary medicine kits were measured before and after sprayed with the three disinfectants (SAEW, KAS and GS), respectively. The sampling methods on the clothing surfaces were same as described above. The percent reduction in microbes was calculated relative to the control using the following Equation (1) ${ }^{[20]}$ :

$$
P=100\left(C_{c}-C_{t}\right) / C_{c}
$$

where, $P$ is percent reduction, $\% ; C_{c}$ is the survival populations of microbes in the control; $C_{t}$ is the survival populations of microbes in the treatment.

\subsection{Inoculation of the samples}

The clothing was obtained from workers in the poultry farm, and the kits made from white cardboard were obtained from the packaging carton of Veterinary medicine (Jindanduo, Zhengda biological technology co., LTD., Zhengzhou, China). In addition, the irons were purchased from a local supermarket in Beijing. All the samples were washed with tap water to remove the soil and then trimmed to approximately $4 \mathrm{~cm} \times 4 \mathrm{~cm}$ in size and packed in a polyethylene bag for the experiment. Before inoculation, samples were inactivated in an autoclave (YXQ-LS-18SI, Shanghai Boxun Industrial Co., Ltd., Shanghai, China) and then air-dried under a biosafety hood (DH-920, Beijing East Union Hall Instrument Manufacturing Co., Ltd., Beijing, China) at room temperature for $50 \mathrm{~min}$ to remove the water. Each sample was inoculated by spreading $0.1 \mathrm{~mL}$ onto the front side region of the prepared culture inoculum with the pipette tip, respectively. Subsequently, all inoculated samples were air-dried under biosafety hood (DH-920, Beijing East Union Hall Instrument Manufacturing Co., Ltd., Beijing, China) for $30 \mathrm{~min}$ at room temperature to allow the bacterial attachment. The final concentration of S. enteritidis inoculated on the clothing, kits and irons was about $6 \log _{10} \mathrm{CFU} / \mathrm{cm}^{2}$ on average. Samples for each treatment were prepared and sampled at least in duplicate.

\subsection{Treatment of samples}

Inoculated samples were sprayed with prepared disinfectants by two automatic sprayers under different conditions (Table 1). Before spraying, the plastic 
buckets were washed by using tap water to remove the former disinfectant. After treatment, moisten sterile swabs with neutralizing agent $\left(0.1 \% \mathrm{Na}_{2} \mathrm{~S}_{2} \mathrm{O}_{3}\right)$ were used to collect the surface microbes. The sterilized cotton swabs, which had been wiped back and forth for twenty times on the sample surfaces, were immediately transferred into $5 \mathrm{~mL}$ neutralizing agent $\left(0.1 \% \mathrm{Na}_{2} \mathrm{~S}_{2} \mathrm{O}_{3}\right)$ tubes for microbiological analyses. The tubes were shaken on a platform shaker at $1800 \mathrm{r} / \mathrm{min}$ (MIR-S100, Sanyo Electric Biomedical Co., Ltd., Osaka, Japan). Surviving bacteria was determined by serial dilutions in sterile $0.1 \%$ peptone water and then plated in duplicate $(0.1 \mathrm{~mL})$ on tryptic soy agar plates. The plates were incubated at $37^{\circ} \mathrm{C}$ for $24 \mathrm{~h}$ to counting of colonies. Moreover, un-inoculated samples yielded no colonies on the agar. Two trials with three replicates in each treatment were done.

\subsection{Model development and statistical analysis}

All treatments were replicated three times and results were reported as means. The value was expressed as the log reductions between final load after the treatments and the initial inoculate per sample. Origin (Version 9.0, OriginLab Cor., Hampton, USA) was used for multiple linear regression analysis and to generate the models. The statistical significance and goodness of fit of the models were evaluated using the determination coefficients $\left(R^{2}\right)$, adjust $R^{2}$. The statistical significance of model was determined using the Fisher $F$-test. The $t$-test was performed to determine significance of differences between model variables. The statistical significance differences between disinfectants were evaluated using the Duncan's multiple range tests.

\section{Results}

\subsection{Reduction in microbes with spraying different disinfectants}

The microbial population on the surfaces of clothing and kits in the disinfection channel is shown in Figure 1, before and after spraying. The initial population on the surfaces of clothing and kits was $1.45 \pm 0.63$ $\log _{10} \mathrm{CFU} / \mathrm{cm}^{2} \quad$ and $2.01 \pm 0.37 \quad \log _{10} \mathrm{CFU} / \mathrm{cm}^{2}$, respectively. The number of microbes on clothing surfaces decreased by $75.3 \% \pm 4.3 \%, 61.5 \% \pm 2.7 \%$ and
$59.7 \% \pm 2.7 \%$ after exposure to SAEW, KAS and GAS. The number of microbial cells on kit surfaces decreased by $72.7 \% \pm 3.8 \%, 58.7 \% \pm 1.3 \%$ and $59.6 \% \pm 2.9 \%$ after exposure to SAEW, KAS and GS. There was no significant difference $(p>0.05)$ in microbial reduction between KAS and GS on clothing and kit surfaces. There were significant differences $(p<0.05$; however, in microbial reduction between SAEW and GS, as well as significant differences $(p<0.05)$ between SAEW and KAS on clothing and kit surfaces.

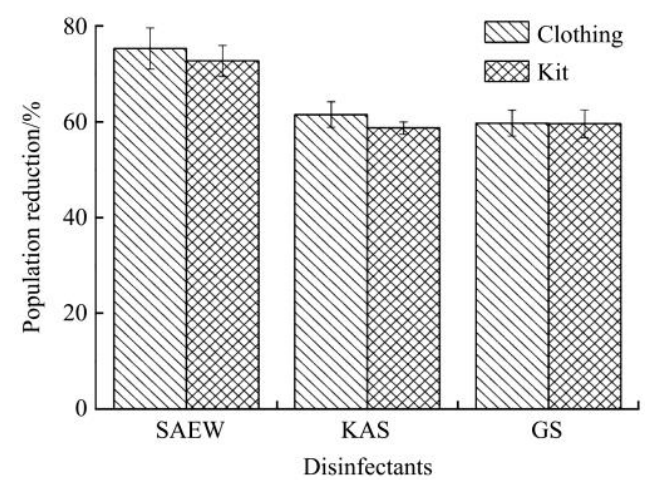

Note: SAEW: slightly acidic electrolyzed water; KAS: Kuei A bromide solution; GS: Glutaraldehyde solution.

Figure 1 Population of microbes on the surfaces of clothing and

kits in disinfection channel before and after disinfection

\subsection{Predictive models}

Multiple linear regression analysis was applied to analyze the influence of treatment time and ACC on the inactivation of $S$. enteritidis in the disinfection channel on the surfaces of clothing, iron materials, and kits. The variables used in the experimental design are listed in Table 2.

Multiple regressions were performed to model the equation. The $Y$ was measured in terms of log reduction. The general model equation was:

$$
Y_{i}=0.216 x_{1}-0.523 x_{2}+0.666 x_{3}+0.006 x_{4}-0.349
$$
where, the $Y_{i}=\log _{10}$ reduction of S. enteritidis. The variables $x_{1}$ and $x_{2}$ were dummy variables determined according to the kinds of materials that were treated. In the model, $x_{1}=x_{2}=1$ when the material is a kit, $x_{1}=1, x_{2}=0$ when the material is an iron material, and $x_{1}=0, x_{2}=1$ when the material is clothing. In addition, $x_{3}$ is the treatment time and $x_{4}$ is the ACC. The analysis of variance (ANOVA) of the quadratic model was performed using the Origin software. The $R^{2}$ value was 0.939 and the adj- $R^{2}$ value was 0.936 respectively, 
indicating that the derived models fit the experimental data well. The statistical significance of the model was determined using the Fisher $F$-test. The analysis of variance for $\log _{10}$ reductions showed that the model equation has a significant effect $(p<0.001)$ on the model prediction, and the regression coefficients $x_{1}, x_{2}, x_{3}$ and $x_{4}$ were significant with small $p$ values $(p<0.001)$.

Table 2 Observed reduction values of $S$. enteritidis on the surface of kit, iron and clothes

\begin{tabular}{|c|c|c|c|c|}
\hline $\begin{array}{l}\text { Time } \\
/ \mathrm{min}\end{array}$ & $\begin{array}{c}\mathrm{ACC} \\
/ \mathrm{mg} \cdot \mathrm{L}^{-1}\end{array}$ & $\begin{array}{c}\text { Kit } \\
\text { Observed value } \\
/ \log _{10} \mathrm{CFU} \cdot \mathrm{cm}^{-2}\end{array}$ & $\begin{array}{c}\text { Iron } \\
\text { Observed value } \\
/ \log _{10} \text { CFU } \cdot \mathrm{cm}^{-2}\end{array}$ & $\begin{array}{c}\text { Clothes } \\
\text { Observed value } \\
/ \log _{10} \mathrm{CFU} \cdot \mathrm{cm}^{-2}\end{array}$ \\
\hline 0.5 & 63 & $0.26 \pm 0.01$ & $0.48 \pm 0.06$ & $0.04 \pm 0.01$ \\
\hline 0.5 & 108 & $0.59 \pm 0.03$ & $0.69 \pm 0.08$ & $0.10 \pm 0.02$ \\
\hline 0.5 & 140 & $0.75 \pm 0.04$ & $0.93 \pm 0.02$ & $0.39 \pm 0.01$ \\
\hline 0.5 & 200 & $1.08 \pm 0.01$ & $1.30 \pm 0.01$ & $0.73 \pm 0.15$ \\
\hline 0.5 & 220 & $1.20 \pm 0.11$ & $1.46 \pm 0.06$ & $1.04 \pm 0.08$ \\
\hline 1 & 63 & $0.56 \pm 0.01$ & $0.86 \pm 0.07$ & $0.18 \pm 0.01$ \\
\hline 1 & 108 & $0.87 \pm 0.06$ & $1.39 \pm 0.07$ & $0.48 \pm 0.03$ \\
\hline 1 & 140 & $0.96 \pm 0.04$ & $1.41 \pm 0.04$ & $0.60 \pm 0.01$ \\
\hline 1 & 200 & $1.15 \pm 0.02$ & $1.63 \pm 0.07$ & $1.01 \pm 0.03$ \\
\hline 1 & 220 & $1.32 \pm 0.07$ & $1.84 \pm 0.04$ & $1.32 \pm 0.03$ \\
\hline 1.5 & 63 & $0.80 \pm 0.01$ & $1.13 \pm 0.12$ & $0.39 \pm 0.01$ \\
\hline 1.5 & 108 & $0.92 \pm 0.13$ & $1.34 \pm 0.15$ & $0.70 \pm 0.05$ \\
\hline 1.5 & 140 & $1.29 \pm 0.04$ & $1.59 \pm 0.10$ & $1.19 \pm 0.10$ \\
\hline 1.5 & 200 & $1.49 \pm 0.04$ & $2.31 \pm 0.02$ & $1.57 \pm 0.06$ \\
\hline 1.5 & 220 & $1.70 \pm 0.05$ & $2.61 \pm 0.04$ & $1.75 \pm 0.08$ \\
\hline 2 & 63 & $1.06 \pm 0.03$ & $1.70 \pm 0.06$ & $0.64 \pm 0.07$ \\
\hline 2 & 108 & $1.24 \pm 0.10$ & $2.01 \pm 0.01$ & $0.87 \pm 0.10$ \\
\hline 2 & 140 & $1.53 \pm 0.01$ & $2.21 \pm 0.02$ & $1.32 \pm 0.10$ \\
\hline 2 & 200 & $1.90 \pm 0.03$ & $2.61 \pm 0.10$ & $1.84 \pm 0.04$ \\
\hline 2 & 220 & $2.01 \pm 0.04$ & $2.61 \pm 0.06$ & $2.02 \pm 0.02$ \\
\hline 2.5 & 63 & $1.46 \pm 0.09$ & $2.61 \pm 0.04$ & $0.85 \pm 0.02$ \\
\hline 2.5 & 108 & $1.59 \pm 0.03$ & $2.61 \pm 0.19$ & $1.37 \pm 0.01$ \\
\hline 2.5 & 140 & $1.74 \pm 0.06$ & $2.61 \pm 0.02$ & $1.73 \pm 0.12$ \\
\hline 2.5 & 200 & $2.24 \pm 0.09$ & $2.61 \pm 0.04$ & $2.23 \pm 0.07$ \\
\hline 2.5 & 220 & $2.36 \pm 0.04$ & $2.61 \pm 0.01$ & $2.36 \pm 0.03$ \\
\hline
\end{tabular}

Table 2 shows the observed values for clothing, iron materials, and kits treated with SAEW for different times at different ACCs. In Figures 2 and 3, the effects of ACC and treatment time on the inactivation of S. enteritidis on kits, iron materials, and clothing are given, respectively. Figure 2 shows the effects of treatment time on the inactivation of $S$. enteritidis on kits, iron materials and clothing. The reduction of S. enteritidis increased as time rose. The reduction in S. enteritidis reached $1.744,2.613$, and 1.734 values for $\log _{10}$ reduction under different conditions of kits, iron materials, and clothing (initial population values were $3.292 \log _{10} \mathrm{CFU} / \mathrm{cm}^{2}, 2.613 \log _{10} \mathrm{CFU} / \mathrm{cm}^{2}$ and 3.103 $\log _{10} \mathrm{CFU} / \mathrm{cm}^{2}$ for controls), respectively. These values were measured at $2.5 \mathrm{~min}$ while keeping the ACC at $140 \mathrm{mg} / \mathrm{L}$.

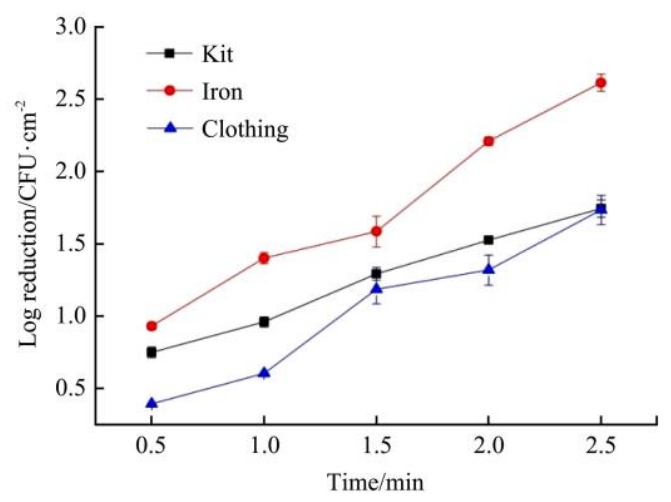

Figure 2 Effects of treatment time on the inactivation of S. enteritidis at ACC of $140 \mathrm{mg} / \mathrm{L}$

The effect of ACC on the inactivation of S. enteritidis on kits, iron materials, and clothing is shown in Figure 3. The reduction of $S$. enteritidis enhanced with increasing ACC. Under the condition where ACC was $220 \mathrm{mg} / \mathrm{L}$ at a constant time of $1 \mathrm{~min}$, the reductions were $1.316 \log _{10}$ $\mathrm{CFU} / \mathrm{cm}^{2}, \quad 1.837 \log _{10} \mathrm{CFU} / \mathrm{cm}^{2}$, and $1.320 \quad \log _{10}$ $\mathrm{CFU} / \mathrm{cm}^{2}$.

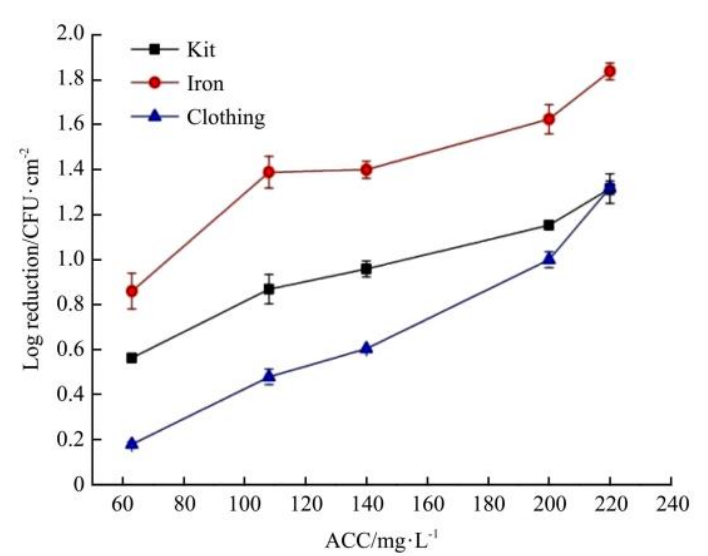

Figure 3 Effects of ACC on the inactivation of S. enteritidis on kit, iron and clothing at treatment time of $1 \mathrm{~min}$

\section{Discussion}

Figure 1 demonstrates that SAEW is an effective sanitizer and an effective approach to reduce the volume of pathogens in the disinfection channel. At an ACC of $220 \mathrm{mg} / \mathrm{L}$, SAEW had a higher inactivation efficiency than $\operatorname{KAS}(5: 1000 \mathrm{v} / \mathrm{v})$ and GS $(5: 1000 \mathrm{v} / \mathrm{v})$. Moreover, during the experimental period, the experimenters were made to cough by the KAS and GS sprayed in the 
disinfection channel, where breeders and other personal must remain for more than $0.5 \mathrm{~min}$ to ensure sterility. Due to its neutral $\mathrm{pH}$, SAEW does not cause coughing as aggressively as KAS and GS, because of the less $\mathrm{Cl}_{2}$ off-gassing ${ }^{[16]}$. Therefore, SAEW is a particularly attractive alternative to KAS and GS for practical use in the disinfection channel.

Figure 2 shows the reduction of S. enteritidis increases as time rises. This result indicates that increasing the contact time during treatment may enhance the effectiveness of SAEW. The workers' stay time in the disinfection channel; however, should not exceed 1 min under two automatic sprayers (droplets: 80-90 $\mu \mathrm{m}$ ), to avoid soaking their clothing. Therefore, it is vital to maintain a short disinfection time for the people who enter the disinfection channel. No data reports have been found that, regarding the amount of time, people should stay in the disinfection channel before farm entry. Thus, future studies should be conducted to confirm the appropriate time for people to stay in the disinfection channel.

Figure 3 shows ACC has a significant effect $(p<0.001)$ on bacterial reduction on kits, iron materials, and clothing surfaces. Similar work was done by Park et al. ${ }^{[22]}$ Several authors have demonstrated that bacterial reduction increases with increasing $\mathrm{ACC}^{[23,24]}$. Moreover, even at equivalent ACCs, SAEW kills microorganisms more quickly than other chemical products, such as sodium hypochlorite $(\mathrm{NaClO})^{[16,19]}$. This may be due to the relative degree to which each chlorine compound is dependent on $\mathrm{pH}$ and temperature $^{[17]}$. The chlorine species present in SAEW at a $\mathrm{pH}$ of 5.0 to 6.5 is hypochlorous acid $(\mathrm{HOCl})$, which is 80 times more effective as a sanitizer than an equivalent concentration of the hypochlorite ion $\left(\mathrm{ClO}^{-}\right)$in inactivating $E$. coli ${ }^{[25]}$. Consequently, ACC is an important factor accounting for bactericidal potency, and may be the primary factor determining the bactericidal activity of SAEW, rather than treatment time. Quan et al. ${ }^{[24]}$ have reported similar results.

The maximum $2.613 \log _{10}$ reduction of bacteria in iron materials (initial populations of $2.613 \log _{10} \mathrm{CFU} / \mathrm{cm}^{2}$ for control) was obtained with ACC $200 \mathrm{mg} / \mathrm{L}$ at a treatment time of 2 min. However, only $2.362 \log _{10}$ and $2.359 \log _{10}$ reductions were observed for kits and clothing surfaces (initial populations of $3.292 \log _{10} \mathrm{CFU} / 16 \mathrm{~cm}^{2}$ and $3.103 \log _{10} \mathrm{CFU} / 16 \mathrm{~cm}^{2}$ ) after treatment with the same SAEW at ACC of $220 \mathrm{mg} / \mathrm{L}$ at a treatment time of 2.5 min (Table 1). In addition, the analysis of virtual variance $x_{1}$, which was the common regression coefficient for kits and iron materials, was significant $(p<0.001)$, and the virtual variance $x_{2}$, the common regression coefficient for kits and clothing, was also greatly $(p<0.001)$. The results shown in Figures 2 and 3 show how the inactivation efficiency of $S$. enteritidis when sprayed by SAEW treatment on iron materials, kits and clothing surfaces were different (iron materials $>$ kits $>$ clothing). Thus, the materials are highly significant factors when designing optimal disinfection using SAEW. Several studies have shown the same results. Liu and $\mathrm{Su}^{[26]}$ found that L. monocytogenes immersed in EO water (50 $\mathrm{mg} / \mathrm{L}$ chlorine) for $5 \mathrm{~min}$ was reduced in number by $3.73 \log _{10} \mathrm{CFU} / 25 \mathrm{~cm}^{2}$ on stainless steel, $4.24 \log _{10}$ CFU/25 $\mathrm{cm}^{2}$ on ceramic tile, and only $1.52 \log _{10}$ $\mathrm{CFU} / 25 \mathrm{~cm}^{2}$ on floor tile. Arevalos-Sánchez et al. ${ }^{[27]}$ reported that material and temperature, as well as material and time of exposure, were two highly significant interaction effects in Neutral electrolyzed water (NEW) used for the reduction of $L$. monocytogenes biofilm populations. Hao et al. ${ }^{[21]}$ also noted that SAEW considerably reduced the number of microbes found on the railings and floors, with the percentage reduction points of $85 \%$ and $81 \%$. They found spraying SAEW on the walls resulted in only a percent reduction of $36 \%$.

\section{Conclusions}

This study demonstrates the effectiveness of using near-neutral electrolyzed water as a microbial decontamination agent on surfaces to reduce bacterial populations. This was shown on kits, iron materials and clothing, and SAEW was compared to KAS and GS. SAEW is significantly effective $(p<0.05)$. It not only reduced bacteria in the disinfection channel, but also prevented potential health hazards to workers due to the lack of $\mathrm{Cl}_{2}$ off-gassing associated with SAEW compared to KAS and GS. Moreover, the established model had a 
good statistical performance, showing an effective function on the treatment time and ACC for predicting reductions in a population of $S$. enteritidis on kits, iron materials and clothing in the disinfection channel.

\section{Acknowledgements}

The author gratefully acknowledges the financial support from China Agricultural Research Systems (CARS-41), Southern plant-eating livestock microclimate regulation technology research (201303145), and Science and Technology Plan Projects of Department of Education of Jiangxi Province (Grant No.: GJJ160401). The author also likes to acknowledge Zhao Lijie for assisting with the experiments.

\section{[References]}

[1] Dorea F C, Berghaus R, Hofacre C, Cole D J. Survey of biosecurity protocols and practices adopted by Growers on Commercial Poultry Farms in Georgia. U.S.A. Avian Diseases, 2010; 54 (3): 1007-1015.

[2] Groseclose S L, Brathwaite W S, Hall P A, Connor F J, Sharp P, Anderson W J, et al. Summary of notifiable diseases-United States. Morb. Mortal. Wkly. Rep., 2002; 51(53): 1-84.

[3] Davies R, Breslin M. Environmental contamination and detection of Salmonella enterica serovar Enteritidis in laying flocks. Vet. Rec, 2001; 149(23): 699-704.

[4] Farber J M. Safe handling of foods. Marcel Dekker, New York, 2000; 41-77.

[5] Baggesen D L, Wingstrand A, Thomsen L K, Mcfadden C, Nielsen B. Salmonella contamination of carcasses from "Salmonella high risk pig pulsed-field gel electrophor-esis". J. Clinical Microbiol, 1997; 36: 2314-2321.

[6] Bialka K L, Demirci A, Knabel S J, Patterson P H, Puri V M. Efficacy of electrolyzed oxidizing water for the microbial safety and quality of eggs. Poultry Science, 2004; 83: 2071-2078.

[7] Dorea F C, Vieira A R, Hofacre C, Waldrip D, Cole D J. Stochastic model of the potential spread of highly pathogenic avian influenza from an infected commercial broiler operation in Georgia. Avian Dis, 2010; 54: 713-719.

[8] Capua I, Marangon S. Control of avian influenza in poultry. Emerg. Infect. Dis., 2006; 12(9): 1319-1324.

[9] Barrington G M, Allen A J, Parish S M, Tibary A. Biosecurity and biocontainment in alpaca operations. Small Rumin. Res., 2006; 61: 217-225.
[10] Gräslund S, Bengtsson B E. Chemicals and biological products used in south-east Asian shrimp farming, and their potential impact on the environment-A review. Sci Total Environ, 2001; 280: 93-131.

[11] Lewis S, McIndoe A K. Cleaning, disinfection and sterilization of equipment. Anaesth Intens Care Med, 2004; 5: $360-363$.

[12] Böhm R. Disinfection and hygiene in the veterinary field and disinfection of animal houses and transport vehicles. Int. Biodeterior Biodegradation, 1998; 41: 217-224.

[13] Zheng W C, Ni L, Hui X, Li B M, Zhang J F. Optimization of slightly acidic electrolyzed water spray for airborne culturable bacteria reduction in animal housing. Int J Agric \& Biol Eng, 2016; 9(4): 185-191.

[14] Huang Y R, Hung Y C, Hsu S Y, Huang Y W, Hwang D F. Application of electrolyzed water in the food industry. Food Contr, 2008; 19: 329-345.

[15] Abadias M, Usall J, Oliveira M, Alegre I, Vinas I. Efficacy of neutral electrolyzed water (NEW) for reducing microbial contamination on minimally processed vegetables. Int. J. Food Microbiol, 2008; 123: 151-158.

[16] Guentzel J L, Lam K L, Callan M A, Emmons S A, Dunham V L. Reduction of bacteria on spinach, lettuce, and surfaces in food service areas using neutral electrolyzed oxidizing water. Food Microbiol, 2008; 25: 36-41.

[17] Koide S, Takeda J, Shi J, Shono H, Atungulu G G. Disinfection efficacy of slightly acidic electrolyzed water on fresh cut cabbage. Food Contr, 2009; 20: 294-297.

[18] Yoshifumi H. Improvement of the electrolysis equipment and application of slightly acidic electrolyzed water for dairy farming. J. Jap. Socie. Agri. Machin., 2003; 65: 27-29.

[19] Cao W, Zhu Z, Shi Z X, Wang C Y, Li B M. Efficiency of slightly acidic electrolyzed water for inactivation of Salmonella enteritidis and its contaminated shell eggs. Int. J. Food Microbiol, 2009; 130: 88-93.

[20] Hao X X, Li B M, Wang C Y, Cao W. Application of slightly acidic electrolyzed water for inactivating microbes in a layer breeding house. Poult. Sci., 2013; 92: 2560-2566.

[21] Hao X X, Li B M, Zhang Q, Lin B Z, Ge L P, Wang C Y, Cao W. Disinfection effectiveness of slightly acidical electrolyzed water in swine barns. J. Appl. Microbiol, 2013; 115: 703-710.

[22] Park H, Hung Y C, Chung D. Effects of chlorine and pH on efficacy of electrolyzed water for inactivating Escherichia coli O157:H7 and Listeria mono-cytogenes. Int. J. Food Microbiol, 2004; 91: 13-18.

[23] Rahman S M E, Ding T, Oh D H. Inactivation effect of newly developed low concentration electrolyzed water and other sanitizers against microorganisms on spinach. Food Contr, 
2010; 21: 1383-1387.

[24] Quan Y, Choi K D, Chung D, Shin I S. Evaluation of bactericidal activity of weakly acidic electrolyzed water (WAEW) against Vibrio vulnificus and Vibrio parahaemolyticus. Int. J. Food Microbiol, 2010; 136: 255-260.

[25] Anonymous. Principle of formation of electrolytic water. Hoshizaki Electric Co. Ltd., Sakae, Toyokae, Aichi, Japan, 1997.
[26] Liu C C, Su Y C. Efficiency of electrolyzed oxidizing water on reducing Listeria monocytogenes contamination on seafood processing gloves. Int. J. Food Microbiol, 2006; 110: 14-15.

[27] Arevalos-Sánchez M, Regalado C, Martin S E, Domínguez-Domínguez J, García-Almendárez B E. Effect of neutral electrolyzed water and nisin on Listeria monocytogenes biofilms, and on listeriolysin $\mathrm{O}$ activity. Food Contr, 2012; 24: 116-122. 